\title{
Hybrid Agents Based Architecture on Automated Dynamic Environments
}

\author{
Dante I. Tapia ${ }^{1}$, Javier Bajo ${ }^{1}$, Juan M. Corchado ${ }^{1}$, Sara Rodríguez ${ }^{1}$ and Juan M. \\ Manzano $^{1}$ \\ ${ }^{1}$ Departamento Informática y Automática, Universidad de Salamanca \\ Plaza de la Merced s/n, 37008, Salamanca, Spain \\ \{dantetapia, jbajope, corchado,srg\}@usal.es,jms.manzano@gmail.com
}

\begin{abstract}
This paper presents a hybrid agents based architecture that uses casebased reasoning and case-based planning systems as reasoning mechanism in deliberative BDI agents to manage daily tasks and hardware devices on dynamic environments. This architecture is also founded on Ambient Intelligence, joining a set of technologies, such as radio frequency identification, wireless networks and wireless control devices, which can be implemented on a wide diversity of environments.
\end{abstract}

Keywords: Hybrid Systems, Case-Based Reasoning, Case-Based Planning, Ambient Intelligence, Automation.

\section{Introduction}

Agents and multi-agent systems (MAS) have become increasingly relevant for developing distributed and dynamic open systems. This paper is focused on describing the main characteristics of an hybrid agents based architecture that use deliberative BDI (Believe, Desire, Intention) agents with Case-Based Reasoning (CBR) and Case-Based Planning (CBP) as reasoning mechanisms as a way to implement adaptive systems on automated dynamic environments. It is also examined Radio Frequency Identification (RFID), wireless networks (Wi-Fi), and ZigBee (802.15.4) devices that can provide the agents real time information about the environment to create plans to manage and automate daily tasks and physical services.

This architecture is also founded on Ambient Intelligence (AmI) environments, characterized by their ubiquity, transparency and intelligence. Ambient Intelligence proposes a new way to interact between people and technology, where this last one is adapted to individuals and their context, showing a vision where people are surrounded by intelligent interfaces merged in daily life objects [9], creating a computing-capable environment with intelligent communication and processing to the service of people by means of a simple, natural, and effortless human-system interaction for users [16], reason why to develop intelligent and intuitive systems and interfaces, capable to recognize and respond to the users necessities in a ubiquous way [8], considering people in the centre of the development [17], and creating 
technologically complex environments in medical, domestic, academic, etc. fields. [19]. Agents in this context must be able to respond to events, take the initiative according to their goals, communicate with other agents, interact with users, and make use of past experiences to find the best plans to achieve goals.

This work is aimed to develop an architecture using hybrid agents, implementing $\mathrm{CBR}$ and $\mathrm{CBP}$ reasoning mechanisms into deliberative BDI agents. CBP reasoning mechanism derivates from CBR systems [1] and are specially designed for planning construction. CBP-BDI facilitates learning and adaptation, and therefore a greater degree of autonomy than that found in pure BDI (Believe, Desire, Intention) architecture [4]. A CBR-BDI agent [6] uses case-based reasoning as a reasoning mechanism, which allows it to learn from initial knowledge, to interact autonomously with the environment as well as with users and other agents within the system, and to have a large capacity for adaptation to the needs of its surroundings. We shall refer to the CBR-BDI agents specialized in generating plans as CBP-BDI agents. BDI agents can be implemented by using different tools, such as Jadex [14]. Jadex agents deal with the concepts of beliefs, goals and plans; they are java objects that can be created and handled within the agent at execution time.

\section{Developing a Hybrid Agents Based Architecture}

The hybrid agents developed are based on the BDI (Belief, Desire, Intention) deliberative architecture model [4], where the internal structure and capabilities of the agents are based on mental aptitudes, using beliefs, desires and intentions. The hybrid architecture model presented in this paper facilitates the incorporation of CBR systems [2] as a deliberative mechanism within BDI agents, facilitating learning and adaptation and providing a greater degree of autonomy than pure BDI architecture. CBR is a type of reasoning based on the use of past experiences [13] to solve new problems by adapting solutions that have been used to solve similar problems in the past, and learn from each new experience. To introduce a CBR motor into a deliberative BDI agent it is necessary to represent the cases used in a CBR system by means of beliefs, desires and intentions, and implement a CBR cycle. The structure of a CBR system is composed of six elements, as seen on figure 1 .

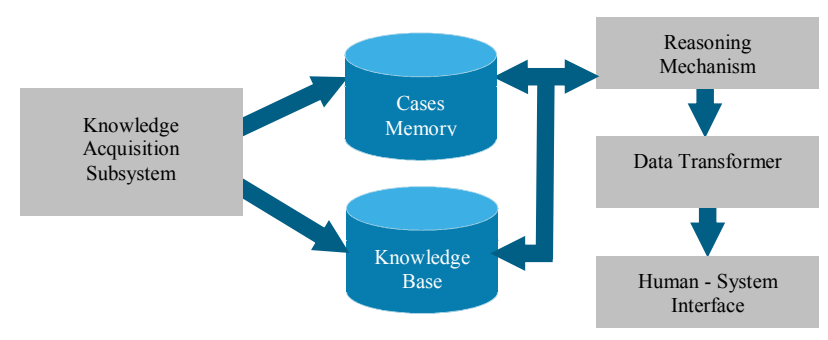

Fig. 1. CBR system structure

The primary concept when working with CBR systems is the concept of case, which is described as a past experience composed of three elements: an initial state or 
problem description that is represented as a belief; a solution, that provides the sequence of actions carried out in order to solve the problem; and a final state that is represented as a set of goals. CBR manages cases (past experiences) to solve new problems. The way cases are managed is known as the CBR cycle, and consists of four sequential phases: retrieve, reuse, revise and retain. The retrieve phase starts when a new problem description is received. Similarity algorithms are applied in order to retrieve from the cases memory the cases with a problem description more similar to the current one. Once the most similar cases have been retrieved, the reuse phase begins, adapting the solutions for the retrieved cases to obtain the best solution for the current case. The revise phase consists of an expert revision of the solution proposed. Finally, the retain phase allows the system to learn from the experiences obtained in the three previous phases and consequently updates the cases memory.

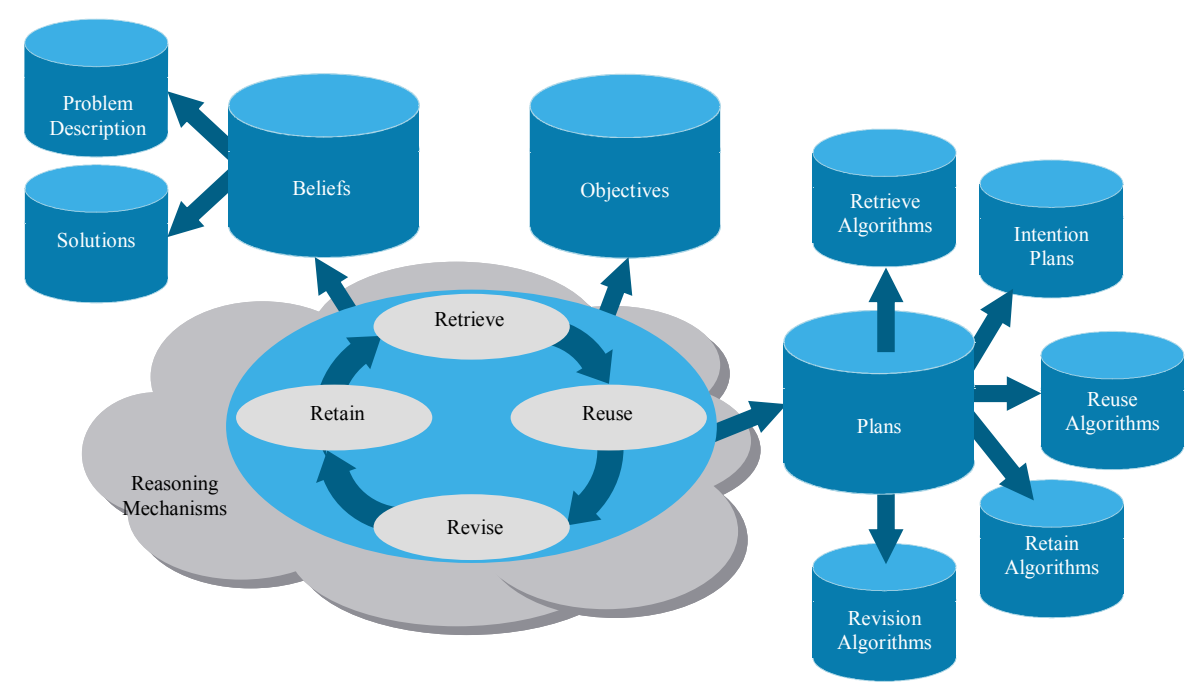

Fig. 2. CBP-BDI deliberative agent internal structure

In a planning agent, the reasoning motor generates plans using past experiences and planning strategies, so the concept of Case Base Planning is obtained [10]. CBP consists of four sequential stages: retrieve stage to recover the most similar past experiences to the current one; reuse stage to combine the retrieved solutions in order to obtain a new optimal solution; revise stage to evaluate the obtained solution; and retain stage to learn from the new experience. Case-based planning (CBP) is the idea of planning as remembering [11]. CBP is a specialization of case-based reasoning (CBR) which is a problem solving methodology based on using a library of solutions for similar problems [11]. In CBP, the solution proposed to solve a given problem is a plan, so this solution is generated taking into account the plans applied to solve similar problems in the past. The problems and their corresponding plans are stored in a plans memory. Figure 2 shows the internal structure of a CPB-BDI agent. Problem description (initial state) and solution (situation when final state is achieved) are 
represented as beliefs, the final state as a goal (or set of goals), and the sequences of actions as plans. The CBP cycle is implemented through goals and plans. When the goal corresponding to one of the stages is triggered, different plans (algorithms) can be executed concurrently to achieve the goal or objective. Each plan can trigger new sub-goals and, consequently, cause the execution of new plans.

\section{Dynamic Environment Automation: Technologies}

The essential aspect in this work is the development of AmI based multi-agent systems that implement hybrid CBR-BDI and CBP-BDI deliberative agents over automated and dynamic environments. Thus the use of technologies that provide the agents automatic and real time information of the context, and allow them to react, is also important. Ambient Intelligence $(\mathrm{AmI})$ provides an effective way to create systems with the ability to adapt themselves to the context and users necessities. The vision of AmI assumes seamless, unobtrusive, and often invisible but also controllable interactions between humans and technology. AmI provides new possibilities for resolving a wide range of problems and proposes a new way to interact between people and technology, where this last one is adapted to individuals and their context, showing a vision where people are surrounded by intelligent interfaces merged in daily life objects [9], creating a computing-capable environment with intelligent communication and processing to the service of people by means of a simple, natural, and effortless human-system interaction for users [16]. One of the most benefited segments of population with the appearance of AmI systems will be the elderly and people with disabilities, improving important aspects of their daily life, especially on health care [9]. Next, three main technologies merged in this architecture are described.

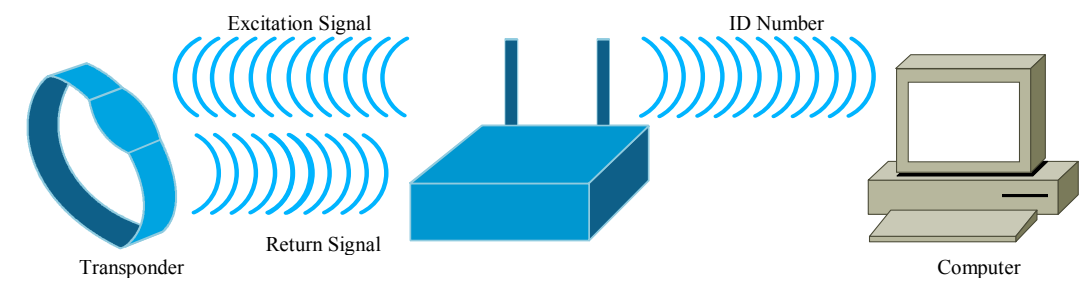

Fig. 3. Functioning of RFID technology

RFID technology is a wireless communications technology used to identify and receive information about humans, animals and objects on the move. An RFID system contains basically four components: tags, readers, antennas and software. Tags with no power system (batteries) integrated are called passive tags or "transponders", these are much smaller and cheaper than tags with some kind of power system included, but have shorter read range. Figure 3 shows how these four elements combined enable the translation of information to a user-friendly format. The transponder is placed on the object itself (i.e. bracelet). As this object moves into the reader's capture area, the 
reader is activated and begins signalling via electromagnetic waves (radio frequency). The transponder subsequently transmits its unique ID information number to the reader, which transmit it to a device or a central computer where the information is processed and showed. This information is not restricted to the location of the object, and can include specific detailed information concerning the object itself. The most use is in industrial/manufacturing, transportation, distribution, etc., but there are other growth sectors including health care [18]. The configuration presented in this paper consists of a transponder mounted on a bracelet worn on the users' wrist or ankle, and several sensors installed over protected zones, with an adjustable capture range up to 2 meters, and a central computer where all the information is processed and stored.

Wireless LANs, also known as Wi-Fi (Wireless Fidelity) networks can be used to replace or as an extension of wired LANs. They provide reduced infrastructure and low installation cost, and also give more mobility and flexibility by allowing people to stay connected to the network as they roam among covered areas, increasing efficiency by allowing data to be entered and accessed on site [12]. The growing use of handheld devices in recent years has led to new necessities as well as to a great opportunity to extend traditional commerce techniques and apply new techniques. These new devices facilitate the use of new interaction techniques, for instance, some systems focus on facilitating users with guidance or location systems [7], [15] by means of their wireless devices. The architecture of the multi-agent system presented in this paper incorporates "lightweight" agents that can reside in mobile devices, such as phones, PDA's, etc. [3], and therefore support wireless communication (Wi-Fi, Bluetooth) which facilitates the portability to a wide range of devices.

ZigBee is a low cost, low power consumption, two-way, wireless communication standard developed by the ZigBee Alliance, based on IEEE 802.15.4 protocol, and operates at $868 / 915 \& 2.4 \mathrm{GHz}$ spectrum. ZigBee is designed to be embedded in consumer electronics, home and building automation, industrial controls, PC peripherals, medical sensor applications, toys and games [20]. This technology is intended for home, building and industrial automation purposes, addressing the needs of monitoring, control and sensory network applications. ZigBee devices can be configured to act as network coordinator (control all devices), as router/repeater (send/receive/resend data to/from coordinator or end devices) or as end device (send/receive data to/from coordinator), connected by star, tree or mesh topologies.

\section{Automated Environment System Prototype}

All the elements described before are integrated into a generic multi-agent system prototype that can be re-modelled to be implemented on different scenarios. First, the essential hardware used and its basic characteristics are presented, and then all the system agents and their main functions are described.

The essential hardware used is: Sokymat's Q5 chip 125KHz RFID wrist bands and computer interface readers for people identification and location monitoring; Silicon Laboratories' C8051 chip-based $2.4 \mathrm{GHz}$ development boards for physical services automation (lights, door locks, alarms, etc.); mobile devices (PDA's) for interfaces and users' interaction; a Workstation where all the high demanding CPU tasks 
(planning and reasoning) are processed; and a basic Wi-Fi network for wireless communication between agents (in PDA's and Workstation). All the hardware is some way integrated to agents, providing them real time information about the environment to create plans to manage and automate daily tasks and physical services.

The system is composed of five different agents, which have specific roles that can be changed according the location and the environment necessities where the system is implemented, for example in geriatric residences [5].

User Agent is a BDI agent that runs on mobile devices (PDA). It manages the users' personal data and behaviour (monitoring, location, daily tasks, and anomalies). The beliefs and goals used for every user depend on the plan or plans defined by the super-users. User Agent maintains continuous communication with the rest of the system agents, especially with the ScheduleUser Agent (through which the scheduledusers can communicate the result of their assigned tasks) and with the SuperUser Agent. The User Agent must ensure that all the actions indicated by the SuperUser are taken out, sending a copy of its memory base (goals and plans) to the Manager Agent in order to maintain backups.

SuperUser Agent is a BDI agent that runs on mobile devices (PDA). It inserts new tasks into the Manager Agent to be processed by the CBR mechanism. It also needs to interact with the User Agents to impose new tasks and receive periodic reports, and with the ScheduleUser Agents to ascertain plans' evolution.

ScheduleUser Agent is a hybrid CBP-BDI planner agent that runs on mobile devices (PDA). It schedules the users' daily activities obtaining dynamic plans depending on the tasks needed for each user. It manages scheduled-users profiles (preferences, habits, holidays, etc.), tasks, available time and resources. Every agent generates personalized plans depending on the scheduled-user profile.

Manager Agent is a hybrid CBR-BDI Agent that runs on a Workstation. It plays two roles: the security role that monitors the users' location trough RFID and manages the ZigBee control devices, and the manager role that handle the medical record database and the tasks assignation. It must provide security for the users and ensure the tasks assignments are efficient. This assignation is carried out through a CBR reasoning engine, which is incorporated within the Manager Agent. When a new assignation of tasks needs to be carried out, both past experiences and the needs of the current situation are recalled, allocating the respective and adequate task.

Devices Agent is a BDI agent that that runs on a Workstation. It monitors the users' location (continuously obtaining/updating data from the RFID readers), and interact with the ZigBee devices to receive information and control physical services (lights, door locks, etc.). The information obtained is sent to the Manager Agent to be processed.

\section{Conclusions and Future Work}

The hybrid architecture presented in this paper solves one of the problems of BDI (deliberative) architectures, which is the lack of learning capacity. The CBR systems reasoning cycle helps the agents to solve problems, adapt to changes in the environment, and identify new possible solutions. 
The use of hybrid agents working in conjunction with RFID, Wireless, and ZigBee technologies, provide a ubiquous, intelligent and adaptable architecture that can be implemented in wide variety scenarios, such as hospitals, geriatric residences, schools, homes or any dynamic environment where is a need to manage tasks and automate services, focusing on security and comfort. In fact, a prototype system, based on this architecture (figure 4), has been successfully implemented into a geriatric residence [5], improving the security and the health care efficiency through monitoring and automating medical staff's work and patients' activities, facilitating the assignation of working shifts and reducing time spent on routine tasks.

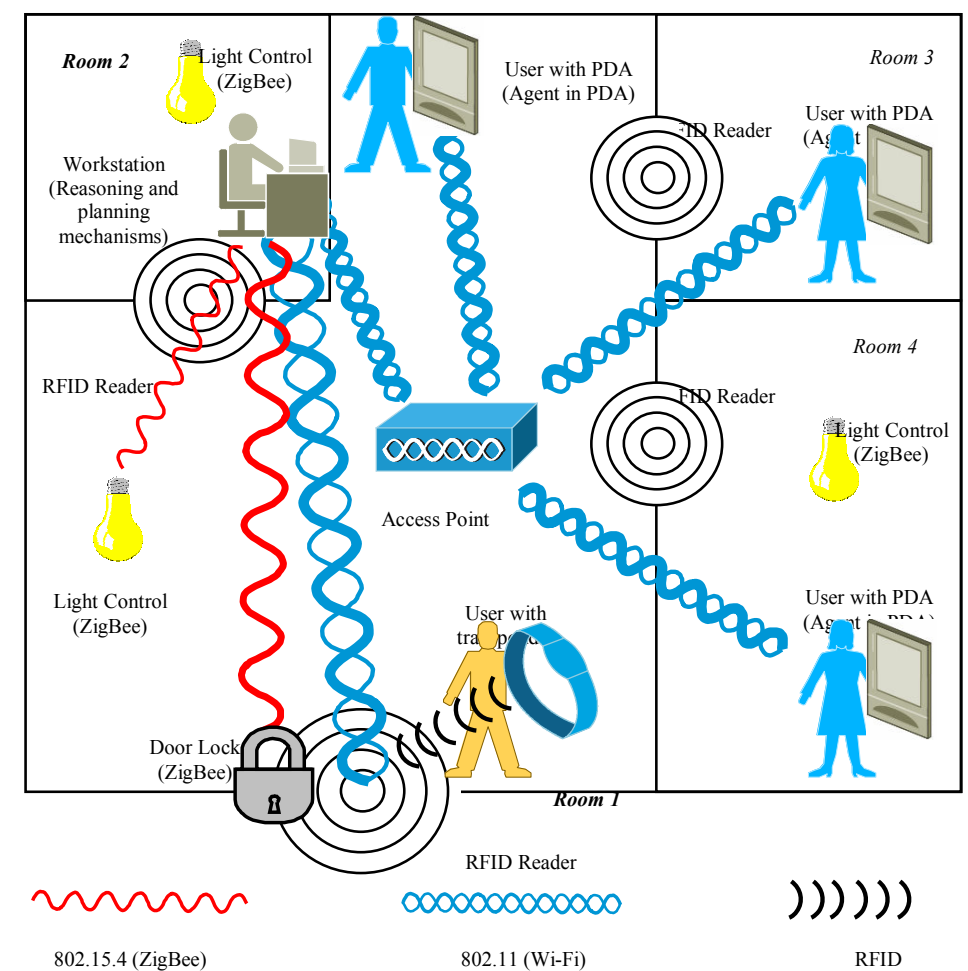

Fig. 4. Architecture applied on an automated environment

CBR-BDI and CBP-BDI agents, working in conjunction with RFID, wireless and automation devices, provide a ubiquous, non-invasive, high level interaction among users and the system, but it is necessary to continue developing and improving the architecture presented, adding new capabilities and integrating new technologies to create flexible and adaptable AmI-based systems to automate services and daily tasks.

Acknowledgements. This work has been partially supported by the MCYT TIN200614630-C03-03. Special thanks to Sokymat 
by the RFID technology provided and to Telefónica Móviles (Movistar) for the wireless devices donated.

\section{References}

1. Aamodt A., Plaza, E.: Case-Based Reasoning: foundational Issues, Methodological Variations, and System Approaches, AICOM. Vol. 7 (1994) 39-59

2. Allen, J.F.: Towards a general theory of action and time. Artificial Intelligence Vol. 23 (1984) 123-154

3. Bohnenberger, T., Jacobs, O., Jameson, A.: DTP meets user requirements: Enhancements and studies of an intelligent shopping guide. Proceedings of the Third International Conference on Pervasive Computing (PERVASIVE-05), Munich, Germany (2005)

4. Bratman, M.E.: Intentions, Plans and Practical Reason. Harvard University Press, Cambridge, M.A (1987)

5. Corchado, J.M., Bajo, J., Tapia, D.I.: ALZ-MAS: Alzheimer's special care multi-agent system. Proceedings of the workshop on Health Care. ECAI'06 (2006)

6. Corchado, J.M., Laza, R.: Constructing Deliberative Agents with Case-based Reasoning Technology. International Journal of Intelligent Systems. Vol. 18 No.12 (2003) 1227-1241

7. Corchado, J.M., Pavón, J., Corchado, E., Castillo, L.F.: Development of CBR-BDI Agents: A Tourist Guide Application. 7th European Conference on Case-based Reasoning 2004. LNAI 3155, Springer Verlag (2005) 547-559

8. Ducatel, K., Bogdanowicz, M., Scapolo, F., Leijten, J., Burgelman, J.C.: That's what friends are for. Ambient Intelligence (AmI) and the IS in 2010. Innovations for an eSociety. Congress Pre-prints, "Innovations for an e-Society. Challenges for Technology Assessment". Berlin, Germany (2001)

9. Emiliani P.L., Stephanidis, C.: Universal access to ambient intelligence environments: opportunities and challenges for people with disabilities. IBM Systems Journal (2005)

10. Glez-Bedia, M., Corchado, J.M.: A planning strategy based on variational calculus for deliberative agents. Computing and Information Systems Journal. Vol.10(1) (2002) 2-14

11. Hammond, K.: Case-Base Planning: Viewing Planning as a Memory Task. Academic Press, New York (1989)

12. Hewlett-Packard.: Understanding Wi-Fi. http://www.hp.com/rnd/library/pdf/ (2002)

13. Kolodner J.: Case-based reasoning. Morgan Kaufmann (1993)

14. Pokahr, A., Braubach L., Lamersdorf, W.: Jadex: Implementing a BDI-Infrastructure for JADE Agents, in: EXP - In Search of Innovation (Special Issue on JADE), Vol. 3 Telecom Italia Lab, Turin, Italy, September (2003) 76-85

15. Poslad, S., Laamanen, H., Malaka, R., Nick, A., Buckle, P., Zipf, A.: Crumpet: Creation of user- friendly mobile services personalised for tourism. In Proceedings of 3G (2001)

16. Richter, K., Hellenschmidt, M.: Interacting with the Ambience: Multimodal Interaction and Ambient Intelligence. Position Paper to the W3C Workshop on Multimodal Interaction, 1920 July (2004)

17. Schmidt, A.: Interactive Context-Aware Systems Interacting with Ambient Intelligence. In G. Riva, F. Vatalaro, F. Davide \& M. Alcañiz, Ambient Intelligence, IOS Press (2005) 159178

18. Sokymat.: Sokymat. http://sokymat.aaitg.com/ (2006)

19. Susperregi, L., Maurtua, I., Tubío, C., Pérez M.A., Segovia, I., Sierra, B.: Una arquitectura multiagente para un Laboratorio de Inteligencia Ambiental en Fabricación. 1er. Taller de Desarrollo de Sistemas Multiagente (DESMA). Málaga, España (2004)

20. ZigBee Standards Organization.: ZigBee Specification Document 053474r13. ZigBee Alliance (2006) 